\title{
Performance and Complexity of Adaptive Lattice Reduction in Fading Channels
}

\author{
Alan T. Murray and Steven R. Weller \\ School of Electrical Engineering and Computer Science \\ University of Newcastle \\ Callaghan, NSW 2308, Australia \\ Email: \{alan.murray,steven.weller\}@newcastle.edu.au
}

\begin{abstract}
Lattice-reduction-aided detection (LRAD) has been shown to considerably increase the performance of linear Multiple-Input Multiple-Output (MIMO) detection systems. In previous work, we introduced Adaptive Lattice Reduction as a way to exploit channel correlation to yield lattice reductionbased detectors exhibiting considerably reduced complexity. In this paper, we review Adaptive Lattice Reduction and examine the performance and complexity of this detection scheme in both temporally and frequency fading channels. For typical mobile environments, for example, we demonstrate a complexity reduction approaching $33 \%$ on a $4 \times 4$ system, without compromising performance.
\end{abstract}

Index Terms-multiple-input multiple-output (MIMO), symbol detection, lattice reduction (LR).

\section{INTRODUCTION}

With increasing sized constellations being used in conjunction with spatial multiplexing Multiple-Input Multiple-Output (MIMO) systems, the complexity of even polynomial-time detection methods can be computationally burdensome [1]. In comparison, linear detection methods increase in complexity linearly proportional to the constellation size and number of transmit antennas used. However, basic linear detection strategies suffer significantly from noise enhancement due to non-orthogonality of the channel matrix.

Lattice-reduction-aided detection (LRAD) has been identified as a way of improving the performance of linear detection strategies. LRAD performs lattice reduction (LR) on the channel matrix to find a more orthogonal set of reduced basis vectors. The Lenstra-Lenstra-Lovász (LLL) algorithm [2] is one such lattice reduction algorithm used to find this set. Performance studies suggest that lattice-reduction-aided detection and derivatives are well suited low complexity solutions when large constellations are used [1], [3].

In this paper we describe the system setup, give a brief overview of LRAD and provide a summary of the LLL algorithm in Sections II, III and IV respectively. We show in Section V that channel correlation can be exploited to yield lattice reduction-based detectors exhibiting considerably reduced complexity. This is achieved via the introduction of a previously introduced technique [4] we call Adaptive Lattice Reduction (ALR). We present results in Section VI which illustrate the extent of this complexity reduction in both temporally and frequency selective fading channels and finally conclude in Section VII.

\section{System Model AND Setup}

We use the complex-valued $N_{\mathrm{t}}$ transmit and $N_{\mathrm{r}}$ receive antenna MIMO model:

$$
\mathbf{y}=\mathbf{H x}+\mathbf{n} .
$$

The $n^{\text {th }}$ transmit antenna is mapped to the constellation set $\mathcal{A}_{n}$ with elements drawn from

$$
\mathbb{X}=\left\{a+i b+\frac{1+i}{2}, a, b \in \mathbb{Z}\right\},
$$

the complex shifted integer grid. The complex vector $\mathbf{x}$ of length $N_{\mathrm{t}}$ represents the transmitted symbols where the element $\mathbf{x}_{n} \in \mathcal{A}_{n}$ is the symbol transmitted from the $n^{\text {th }}$ antenna. Some (but not all) constellations can be considered a subset of $\mathbb{X}$. For example, rectangular constellations such as QPSK or $m$-QAM can be considered a subset of $\mathbb{X}$ but not circular constellations such as 8-PSK.

The channel is modelled by $\mathbf{H}$, an $N_{\mathrm{r}}$ by $N_{\mathrm{t}}$ complex-valued matrix representing the overall channel and has elements $h_{m, n}$ complex-valued non-dispersive fading coefficients of the channel between the $n^{\text {th }}$ transmit and the $m^{\text {th }}$ receive antenna. Gaussian noise is represented by the length $N_{\mathrm{r}}$ vector $\mathbf{n}$ with $\mathbb{E}\{|\mathbf{n}|\}=0$ and $\mathbb{E}\left\{\left|\mathbf{n} \mathbf{n}^{\mathrm{H}}\right|\right\}=\sigma^{2}$. Finally, y represents the vector of received symbols of length $N_{\mathrm{r}}$.

Many works use the real decomposition of the complexvalued MIMO transmission model [5], [6]. Lattice reduction methods can operate on both real and complex integer lattices and in particular the LLL algorithm has been extended for complex lattice reduction [7].

Maximum-likelihood (ML) detection finds the vector of symbol estimates $\widehat{\mathbf{x}}$ which minimises the euclidean distance problem as follows:

$$
\widehat{\mathbf{x}}=\underset{\mathbf{x} \in \mathcal{A}^{N_{\mathrm{t}}}}{\arg \min }\|\mathbf{y}-\mathbf{H x}\|^{2} .
$$

This problem is NP-hard, and the complexity is exponentially proportional to the constellation set size and number of transmit antennas. Various heuristic approaches exist which approximately solve this problem in less than exponential time. Some detectors, such as sphere detectors, typically have complexity subexponentially proportional to the constellation set size and number of antennas and whilst their complexity is 
suitably low for small constellations and low numbers of transmit antennas, their complexity can become considerable as either increase. Linear detection approaches have complexity proportional to the number of transmit antennas only. Linear detection approaches use the inverse of the channel to generate an estimate of the transmitted symbols.

Zero Forcing (ZF) is a linear detection method which estimates $\mathbf{x}$ by finding the least squares solution to (1). However, correlation between the columns of $\mathbf{H}$ results in significant noise enhancement during quantisation of the least squares solution.

\section{Lattice Reduction Aided Detection (LRAD)}

The channel matrix $\mathbf{H}$ can be considered a generator matrix for the lattice $\mathbf{H X}^{N_{t}}$ of which the received symbols are elements. As such, MIMO detection can be considered equivalent to the closest lattice point problem. From lattice theory, methods exist which attempt to reduce noise enhancement. Lattice basis reduction $[8, \S 2.6 .1]$ does this by finding a more orthogonal set of basis vectors using the following steps adapted from [6] and detailed in [9]:

1) Find reduced lattice basis

2) Use pseudoinverse of reduced basis to form estimates

3) Quantise estimates to $\mathbb{X}$

4) Transform result to find transmitted constellation points

The reduced lattice basis is found by optimising the generating matrix, in this case the channel matrix. Complex integer linear combinations of the column vectors of $\mathbf{H}$ are taken to form the closer to orthogonal reduced matrix $\mathbf{H}_{\mathrm{L}}$ which spans the same set of points $\mathbf{H} \mathbb{X}^{N_{t}} \equiv \mathbf{H}_{\mathrm{L}} \mathbb{X}^{N_{t}}$ and so

$$
\mathbf{H}_{\mathrm{L}}=\mathbf{H T} \text { or } \mathbf{H}=\mathbf{H}_{\mathrm{L}} \mathbf{T}^{-1},
$$

where $\mathbf{T}$ is a unimodular matrix with complex integer entries and $\operatorname{det}(\mathbf{T})= \pm 1$, therefore $\mathbf{T}^{-1}$ also contains only complex integer entries.

As in [6], by finding an equivalent and closer to orthogonal set of the basis vectors, $\mathbf{H}_{\mathrm{L}}$, noise enhancement is reduced when quantisation is performed. Importantly, as $\mathbf{T}^{-1}$ and $\mathbf{x}$ both contain only integer spaced entries, so does $\mathbf{T}^{-1} \mathbf{X}$ and so symbol detection or quantisation is merely rounding to the grid $\mathbb{X}$.

We can quantitatively measure the orthogonality of a matrix using metrics such as condition number or orthogonality defect. As in $[10, \S 4.6 .2]$, we measure the orthogonality defect of matrices using:

$$
\delta(\mathbf{H})=\frac{\prod_{k=1}^{N_{\mathrm{t}}}\left\|\mathbf{h}_{k}\right\|}{|\operatorname{det}(\mathbf{H})|},
$$

where $\mathbf{h}_{k}$ is the $k^{\text {th }}$ column of $\mathbf{H}, \delta(\mathbf{H}) \geq 1$ for all $\mathbf{H}$ and $\delta(\mathbf{H})=1$ if and only if the columns of $\mathbf{H}$ are orthogonal.

To assess the impact of lattice redution on orthogonality defect, we generated $10^{6}$ randomly chosen $\mathbf{H} \in \mathbb{C}^{4 \times 4}$, and computed the lattice reduced $\mathbf{H}_{\mathrm{L}}$. The method used to perform lattice reduction is detailed in Section IV. The orthogonality defect was found using (5) before and after lattice reduction.
This gives a quantifiable method to examine the performance of lattice reduction. The result, as shown in Fig. 1, is a considerable reduction in orthogonality defect. It is this improvement that reduces noise enhancement in linear detection methods and reduces the error rate of LRAD based systems.

\section{Lenstra-Lenstra-Lovász (LLL) ALgorithm}

In this section we review the description of a complex LLL reduction algorithm [7]. We extend this description with the purpose of bringing context to enhancement that will follow in Section V. We define:

- $\mathcal{H}_{i}$ as the squared euclidean norm of the orthogonal vectors produced by the Gram-Schmidt Orthogonalisation (GSO) of $\mathbf{H}$.

- $\mu_{i j}$ as the ratio of the length of the orthogonal projection of the $i^{\text {th }}$ basis onto the $j^{\text {th }}$ orthogonal vector and the length of the $j^{\text {th }}$ orthogonal vector.

- $\mathbf{H}_{\mathrm{L}}^{i}$ and $\mathbf{T}^{i}$ represent the values of the reduced basis and transform after the $i^{\text {th }}$ step of the LLL algorithm

- Initially, $\mathbf{H}_{\mathrm{L}}^{0}=\mathbf{H}$ and $\mathbf{T}^{0}=\mathbf{I}_{N_{\mathrm{t}}}$

- $k$ is the index of the current column of $\mathbf{H}$ being processed such that $2 \leq k \leq N_{\mathrm{t}}$

The LLL algorithm then consists of three steps:

1) $\mathcal{H}$ and $\mu$ are computed using a modified GSO procedure [11]

2) Size reduction aims to make basis vectors shorter and more orthogonal by asserting the condition that $\left|\operatorname{Re}\left(\mu_{k, j}\right)\right| \leq 0.5$ and $\left|\operatorname{Im}\left(\mu_{k, j}\right)\right| \leq 0.5$ for all $j<k$

3) Basis vectors $\mathbf{h}_{k-1}$ and $\mathbf{h}_{k}$ are swapped if some condition is satisfied such that size reduction can be repeated to make basis vectors shorter

Size reduction and basis vector swapping iterates until the swapping condition is not satisfied by any pair of $\mathbf{h}_{k-1}$ and $\mathbf{h}_{k}$. The resultant basis is then said to be reduced. The swapping condition for LLL reduction, also called the Lovász condition, is:

$$
\mathcal{H}_{k}<\left(\delta-\left|\mu_{k, k-1}\right|^{2}\right) \mathcal{H}_{k-1},
$$

where $\delta$ with $\frac{1}{4}<\delta<1$ is a factor selected to achieve a good quality-complexity trade off [2].

After each swapping step, $\mathcal{H}_{k-1}, \mathcal{H}_{k}$ and some of the $\mu_{i, j}$ values needed to be updated. Techniques can be employed to minimise the number and frequency of recalculations of $\mathcal{H}$ and $\mu$ elements [11].

\section{COMPlexity Reduction}

Considerable complexity reduction can be achieved in fading channels through the technique we call Adaptive Lattice Reduction (ALR). In a slowly fading channel, the elements of $\mathbf{H}$ change slowly and are therefore correlated in time. It has been demonstrated that for these channels the transform matrix $\mathbf{T}$ is constant for several renditions of the channel [4]. As such, a complexity reduction can be achieved by not directly recalculating the reduced basis $\mathbf{H}_{\mathrm{L}}$ every time the channel varies. 


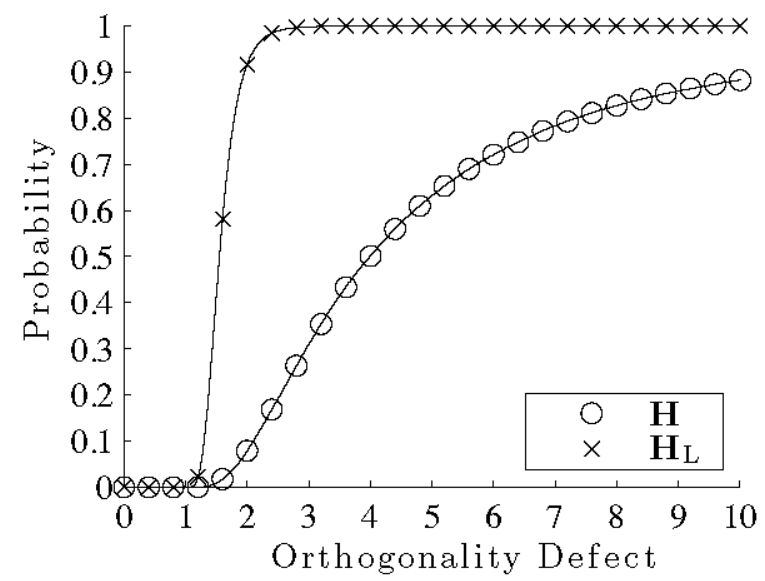

Fig. 1. Cumulative density of the orthogonality defect for non-reduced and reduced basis channel matrices

From (4) we can calculate the new reduced basis by simply multiplying a new $\mathbf{H}$ by a previously calculated $\mathbf{T}$. Even if $\mathbf{T}$ is not the optimal basis transform, the reduced basis still spans the same space as the new $\mathbf{H}$, it is simply not as orthogonal.

Furthermore, as (4) holds true after every step of the LLL algorithm, further complexity reduction can be achieved by using a previously calculated $\mathbf{T}$ to "pre-reduce" a new $\mathbf{H}$ in order to reduce the number of steps required to compute the new reduced basis. We can simply pre-reduce a new $\mathbf{H}$ by calculating $\mathbf{H}^{\prime}=\mathbf{H} \mathbf{T}^{\mathrm{A}}$, where $\mathbf{T}^{\mathrm{A}}$ is a previously calculated basis transform. The aim is that this pre-reduced basis is already closer to orthogonal and many steps of the LLL algorithm can be skipped.

To generate the new reduced basis and transform, we perform lattice reduction on the pre-reduced matrix $\mathbf{H}^{\prime}$ to calculate $\mathbf{H}_{\mathrm{L}}^{\prime}$. This procedure calculates a new basis transform $\mathbf{T}^{\mathrm{B}}$ such that $\mathbf{H}_{\mathrm{L}}^{\prime}=\left(\mathbf{H T}^{\mathrm{A}}\right) \mathbf{T}^{\mathrm{B}}$. The two transforms can be combined to calculate the transform $\mathbf{T}=\mathbf{T}^{\mathrm{A}} \mathbf{T}^{\mathrm{B}}$ such that

$$
\mathbf{H}_{\mathrm{L}}^{\prime}=\mathbf{H T}
$$

$\mathbf{T}$ can then be used to pre-reduce the next $\mathbf{H}$. Alternatively, if the initial value $\mathbf{T}^{0}$ is initialised to the previous transform value $\mathbf{T}^{\mathrm{A}}$, the transform produced by the LLL algorithm will already be $\mathbf{T}$ and the explicit multiplication $\mathbf{T}=\mathbf{T}^{\mathrm{A}} \mathbf{T}^{\mathrm{B}}$ can be skipped.

Finally, again as (4) holds true after every step of the LLL algorithm, the algorithm can be terminated after a predetermined maximum number of steps. This is important as it can be used to constrain the complexity of the algorithm. This would be particularly relevant for a hardware implementation. By combining pre-reduction and early termination, the algorithm will progressively move toward the optimum reduced basis whilst capping the number of iterations performed on each rendition of $\mathbf{H}$.

\section{RESULTS}

Simulations have shown that progressively updating the transform matrix by using this pre-reduction technique yields a significant reduction of the number of steps needed in the LLL algorithm.

\section{A. Temporally Fading Channels}

1) Setup: We reuse the setup from Fig. 1, except this time make use of a time fading channel. We selected to base our simulations around specifications similar to that used in mobile 3GPP environments. Our simulations were developed using a freely available library of mathematical, signal processing and communication routines which includes a substantial multipath fading channel simulator and common COST and ITU channel models [12]. These parameters are outlined in Table I.

TABLE I

TEMPoral FAding Simulation PARAMETERS

\begin{tabular}{|c|c|}
\hline Property & Value \\
\hline Tx / Rx Antennas & $4 \times 4$ \\
Fading Type & Jakes Doppler Spectrum \\
Fading Method & Rice Method of Exact Doppler Spread \\
Carrier Frequency & $2100 \mathrm{MHz}$ \\
Sampling Frequency & $1.92 \mathrm{MHz}$ \\
Fading Period & 960 received symbols \\
& successive blocks are uncorrelated. \\
\hline
\end{tabular}

The sampling frequency selected represents the slowest sampling frequency available in $3 \mathrm{GPP}$ systems. This causes the highest normalised Doppler frequency and as such can be considered a worst case.

2) Quality: We varied the maximum Doppler velocity from $1 \mathrm{~km} / \mathrm{h}$ up to the maximum 3GPP Long Term Evolution target of $350 \mathrm{~km} / \mathrm{h}$ and examined the orthogonality defect of each channel basis before reduction, after lattice reduction and after adaptive lattice reduction. As expected, the cumulative density of the orthogonality defect did not vary as the velocity was increased. Significantly, neither did that of the reduced and adaptively reduced channel basis.

Fig. 2 shows that the cumulative density of the basis orthogonality defect is indistinguishable between the adaptive and unmodified reduction implementations. This confirms that the quality of bases are independent of the maximum Doppler velocity, an intuitively appealing result as the expectation of each individual channel basis is not perturbed by the introduced correlation. Therefore, it can be expected that for a given channel model, adaptive lattice reduction will offer equivalent performance at lower complexity.

3) Complexity: In order to perform a complexity comparison, we counted the number of floating point operations (FLOPS) performed by the LLL algorithm with complex additions counting as 2 and complex multiplications as 6 operations. To provide a fair comparison, we also included the cost of the pre-reduction matrix-matrix multiplication used by adaptive lattice reduction. It should be noted that the complexity of this multiplication accounts for 480 FLOPS or more than half of the complexity of adaptive lattice reduction. The results proved interesting as the complexity of the adaptive scheme did not increase considerably even as the maximum Doppler velocity was increased. This is shown in Table II where the 


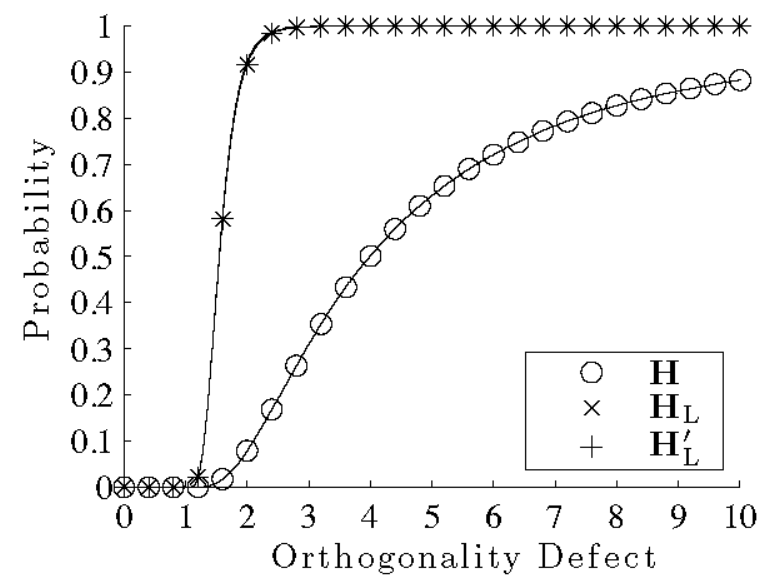

Fig. 2. Cumulative density of the orthogonality defect for a correlated fading channel

percentage of saved FLOPS does not vary considerably, even at the high velocity of $350 \mathrm{~km} / \mathrm{h}$.

TABLE II

COMPLEXITY COMPARISON BETWEEN ADAPTIVE AND NON-ADAPTIVE LATTICE REDUCTION AS THE MAXIMUM DOPPLER VELOCITY IS VARIED.

\begin{tabular}{|c||c|c|c|}
\hline \multicolumn{1}{|c||}{\multirow{2}{*}{$\begin{array}{c}\text { Velocity } \\
(\mathrm{km} / \mathrm{h})\end{array}$}} & \multicolumn{2}{c|}{ FLOPS } & \multirow{2}{*}{ \% saved } \\
\cline { 2 - 3 } & LR & ALR & \\
\hline 10 & 1209 & 821 & $32.1 \%$ \\
60 & 1210 & 821 & $32.1 \%$ \\
120 & 1207 & 821 & $32.0 \%$ \\
350 & 1207 & 821 & $31.9 \%$ \\
\hline
\end{tabular}

In order to find the limiting case of the adaptive scheme, we examined the complexity as the channel update frequency was reduced. This was performed by increasing the number of received symbols between successive channel updates, or in other words by holding the measured value of $\mathbf{H}$ static for several received symbols. This effectively increases the normalised Doppler frequency as seen by the channel reduction algorithm. This simulation was run at the maximum $3 \mathrm{GPP}$ target of $350 \mathrm{~km} / \mathrm{h}$. As can be shown in Fig. 3, the complexity of the adaptive scheme slowly approaches that of the nonadaptive scheme.

4) Performance: In order to compare the error rate performance of adaptive and non-adaptive lattice reduction we chose to implement Subspace Lattice Reduction Aided Detection (SLRAD) [13]. This particular detector was chosen due to its ability to produce a candidate list suitable for generating soft information and close to ML performance. Adaptive Lattice Reduction only varies the complexity of the lattice reduction component of the detector so whilst SLRAD indeed includes components with complexity not examined, this complexity is equal between adaptive and non-adaptive detection. We used the simulation parameters from Table II and measured the bit error rate performance when QPSK was used for modulation.

The performance of both adaptive and non-adaptive SLRAD was shown to be very close to the performance of ML detection, as Fig. 4 illustrates. This confirms our statement

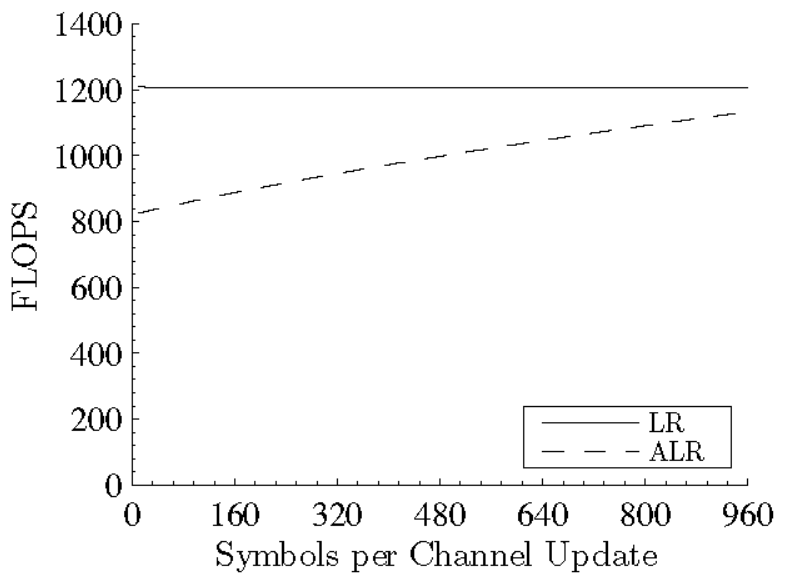

Fig. 3. Complexity comparison between adaptive and non-adaptive lattice reduction as the number of symbols between channel updates is varied.

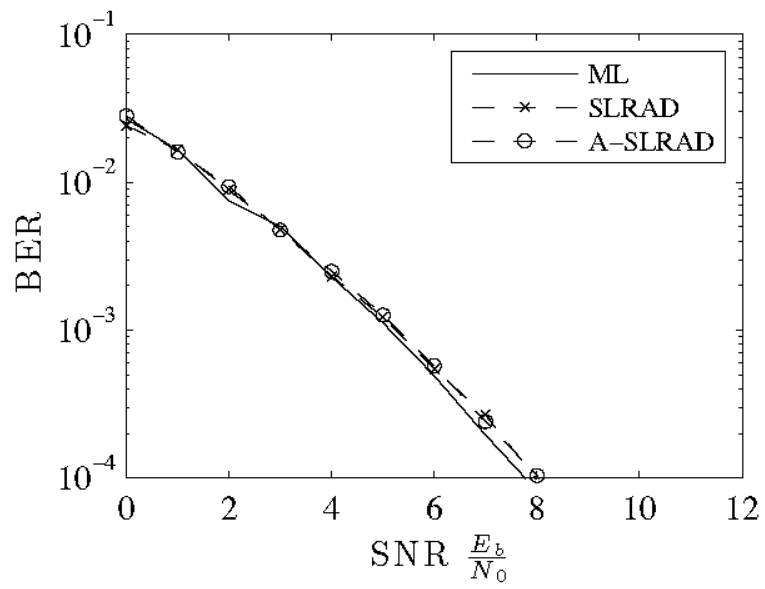

Fig. 4. Performance comparison between adaptive and non-adaptive lattice reduction at $350 \mathrm{~km} / \mathrm{h}$.

from Section VI-A2 that as adaptive and non-adaptive lattice reduction generates bases with equivalent distributions of orthogonality defect, the performance of detectors implementing either method will also be equivalent.

We also examined the performance of SLRAD and ML detection as the channel update frequency was reduced. As with Fig. 3 we increased the number of received symbols between successive channel updates. Fig. 5 shows how the performance of ML and both SLRAD based implementations deteriorated as the update frequency was reduced. Notably, the adaptive scheme matched the performance of the non-adaptive scheme, again as predicted in Section VI-A2. Furthermore, we can see that the performance of all three detection methods has deteriorated significantly when the channel is updated only every 200 received symbols. At this point, the adaptive scheme still offers a significant complexity reduction as shown previously in Section VI-A3.

\section{B. Frequency Selective Fading Channels}




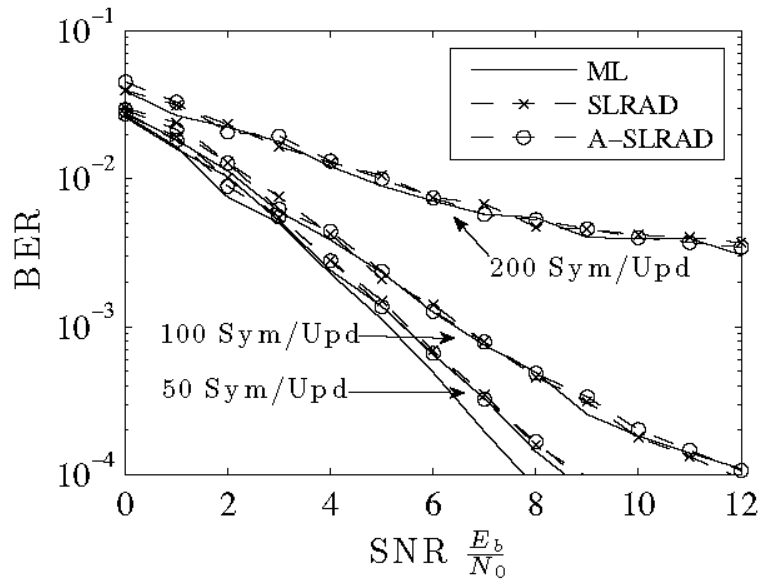

Fig. 5. Performance comparison between adaptive and non-adaptive lattice reduction at $350 \mathrm{~km} / \mathrm{h}$ as the number of symbols between channel updates is varied.

1) Setup: A Frequency selective fading channel can be considered as a set of correlated narrow-band frequency-flat fading channels. In systems employing Orthogonal Frequency Division Multiplexing (OFDM), multipath causes correlated fading across the narrow-band subcarriers of which modulated symbols are transmitted and received. We can therefore examine the performance of Adaptive Lattice Reduction in such channels. Rather than simulating a full OFDM system, we instead take the Fourier Transform of the channel impulse response. This gives us a correlated fading envelope and allows the examination of the frequency domain response of differing multipath channels. As such we implicitly presume that an appropriately long cyclic prefix has been chosen to avoid both Inter-Symbol Interference (ISI) and Inter-Carrier Interference (ICI). These parameters are outlined in Table III.

TABLE III

Frequency Selective FAdING Simulation Parameters

\begin{tabular}{|c|c|}
\hline Property & Value \\
\hline Tx / Rx Antennas & $4 \times 4$ \\
Fading Type & Tapped Delay Line \\
Fading Method & Frequency Response \\
Channel Profile & Various ITU and COST multipath profiles \\
Carrier Frequency & $2100 \mathrm{MHz}$ \\
Sampling Frequency & $30.88 \mathrm{MHz}$ \\
Subcarriers / Tones & 2048 \\
& I.I.D. Fading per multipath \\
\hline
\end{tabular}

2) Complexity: Using the same approach from Section VI-A3, we measured the complexity of adaptive and nonadaptive lattice reduction for various multipath channels. There is a reasonable correlation between the RMS Delay Spread of the channel impulse response and the complexity of the adaptive scheme, as shown in Table IV.

Significant complexity reduction can be achieved in channels with a relatively minimal RMS Delay Spread, or in "Rural Area" (RA) COST profiles. As the RMS Delay Spread increases with the "Typical Urban" (TU) profiles, the com-

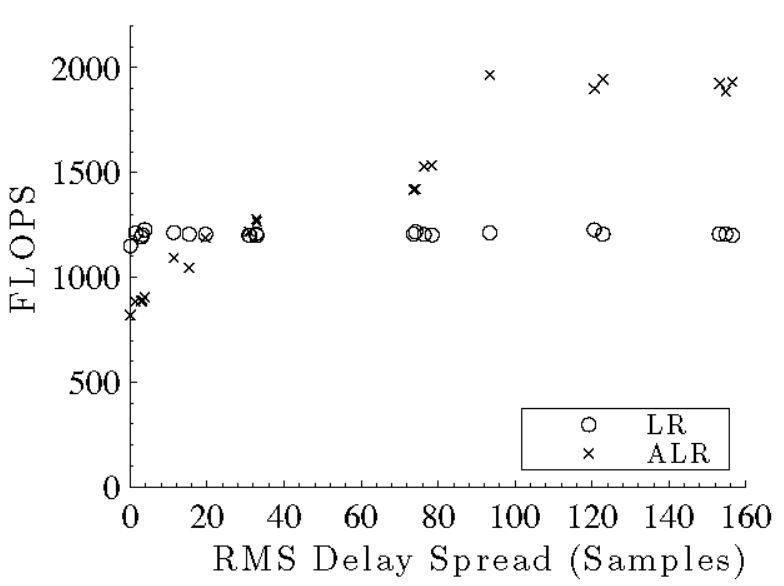

Fig. 6. Complexity comparison between adaptive and non-adaptive lattice reduction for different multipath channels.

plexity of adaptive scheme aproaches and then begins to exceed that of the non-adaptive scheme. In channels with moderate spread, or "Bad Urban" (BU) profiles, the adaptive scheme's complexity further exceeds that of the non-adaptive scheme. Finally, channels with a delay spread over 80 samples, or "Hilly Terrain" (HT) profiles, sees the adaptive scheme's complexity reduction significantly exceed that of the nonadaptive scheme.

We can see the correlation between RMS Delay Spread and Complexity in Fig. 6. Each of the channel profiles is represented by a marker for each scheme. As expected the complexity of non-adaptive lattice reduction is relatively flat, whereas the complexity of adaptive lattice reduction is roughly dependant on RMS Delay Spread.

TABLE IV

COMPLEXITY COMPARISON BETWEEN ADAPTIVE AND NON-ADAPTIVE LATTICE REDUCTION FOR DIFFERENT MULTIPATH CHANNELS.

\begin{tabular}{|c|c|c|c|c|}
\hline \multirow[t]{2}{*}{ Channel } & \multirow{2}{*}{$\begin{array}{c}\text { RMS Delay } \\
\text { Spread (Samples) }\end{array}$} & \multicolumn{2}{|c|}{ FLOPS } & \multirow[t]{2}{*}{$\%$ saved } \\
\hline & & LR & ALR & \\
\hline Delta & 0 & 1150 & 820 & $28.7 \%$ \\
\hline ITU Pedestrian A & 1 & 1212 & 883 & $27.1 \%$ \\
\hline COST207 RA6 & 3 & 1192 & 889 & $25.4 \%$ \\
\hline $\operatorname{cosT} 259 \mathrm{RAx}$ & 3 & 1203 & 890 & $26.0 \%$ \\
\hline COST207 RA & 4 & 1227 & 906 & $26.2 \%$ \\
\hline ITU Vehicular A & 11 & 1215 & 1093 & $10.1 \%$ \\
\hline $\cos 259 \mathrm{TUx}$ & 15 & 1207 & 1046 & $13.4 \%$ \\
\hline ITU Pedestrian B & 20 & 1206 & 1190 & $1.3 \%$ \\
\hline COST207 TU12 & 31 & 1203 & 1219 & $-1.4 \%$ \\
\hline COST207 TU12alt & 31 & 1198 & 1217 & $-1.6 \%$ \\
\hline COST207 TU6alt & 33 & 1209 & 1279 & $-5.8 \%$ \\
\hline COST207 TU & 33 & 1200 & 1267 & $-5.6 \%$ \\
\hline $\cos 2207 \mathrm{BU}$ & 74 & 1207 & 1418 & $-17.5 \%$ \\
\hline COST207 BU6alt & 74 & 1217 & 1423 & $-17.0 \%$ \\
\hline COST207 BU12 & 76 & 1206 & 1529 & $-26.9 \%$ \\
\hline COST207 BU12alt & 78 & 1202 & 1534 & $-27.6 \%$ \\
\hline COST259 HTx & 93 & 1214 & 1965 & $-61.9 \%$ \\
\hline COST207 HT6alt & 121 & 1227 & 1901 & $-54.9 \%$ \\
\hline ITU Vehicular B & 123 & 1206 & 1943 & $-61.2 \%$ \\
\hline COST207 HT12 & 153 & 1208 & 1925 & $-59.4 \%$ \\
\hline COST207 HT & 155 & 1207 & 1886 & $-56.3 \%$ \\
\hline COST207 HT12alt & 157 & 1201 & 1933 & $-60.9 \%$ \\
\hline
\end{tabular}




\section{CONCLUSION}

In this paper we have examined the complexity and performance of Adaptive Lattice Reduction in various temporally and frequency selective fading channels. We have shown that substantial complexity reduction can generally be achieved by exploiting the static nature of the LRAD transform matrix in various fading channels. It has been confirmed through realistic simulations that Adaptive Lattice Reduction can be used without compromising performance. Adaptive Lattice Reduction has considerable potential for improving the performance and reducing complexity in future practical implementations of LRAD based detection schemes for MIMO communication systems.

\section{REFERENCES}

[1] C. Windpassinger, L. Lampe, R. F. H. Fischer, and T. Hehn, "A performance study of MIMO detectors," IEEE Trans. Wireless Commun., vol. 5, no. 8, pp. 2004-2008, Aug. 2006.

[2] A. K. Lenstra, H. W. Lenstra, and L. Lovász, "Factoring polynomials with rational coefficients," Math. Ann., vol. 261, no. 4, pp. 515-534, Dec. 1982

[3] X. Ma and W. Zhang, "Performance analysis for MIMO systems with lattice-reduction aided linear equalization," IEEE Trans. Commun., vol. 56, no. 2, pp. 309-318, Feb. 2008
[4] A. T. Murray and S. R. Weller, "Enhanced lattice-reduction aided detection for MIMO systems," University of Newcastle, Australia, Tech. Rep. SPM386, mar 2008. [Online]. Available: http://sigpromu.org/publications.html

[5] P. Silvola, K. Hooli, and M. Juntti, "Suboptimal soft-output MAP detector with lattice reduction," IEEE Signal Process. Lett., vol. 13 , no. 6, pp. 321-324, Jun. 2006.

[6] C. Windpassinger and R. F. H. Fischer, "Low-complexity nearmaximum-likelihood detection and precoding for MIMO systems using lattice reduction," in Proc. IEEE Information Theory Workshop, Paris, France, Mar. 2003, pp. 345-348.

[7] Y. H. Gan and W. H. Mow, "Complex lattice reduction algorithms for low-complexity MIMO detection," in Proc. IEEE GLOBECOM '05, St. Louis, Missouri, USA, 2005, pp. 2953-2957.

[8] H. Cohen, A Course in Computational Algebraic Number Theory. Berlin, Germany: Springer-Verlag, 1993.

[9] E. Agrell, T. Eriksson, A. Vardy, and K. Zeger, "Closest point search in lattices," IEEE Trans. Inf. Theory, vol. 48, no. 8, pp. 2953-2957, Aug. 2002.

[10] C. Windpassinger, "Detection and precoding for multiple input multiple output channels," Ph.D. dissertation, Universität Erlangen-Nürnberg, Germany, 2004.

[11] W. H. Mow, "Universal lattice decoding: principle and recent advances," Wirel. Commun. Mob. Comput, vol. 3, no. 5, pp. 553-569, Aug. 2003.

[12] ITH - C++ library of mathematical, signal processing and communication routines. [Online]. Available: http://itpp.sourceforge.net

[13] C. Windpassinger, L. H. J. Lampe, and R. F. H. Fischer, "From lattice-reduction-aided detection towards maximum-likelihood detection in MIMO systems," in Proc. WOC 2003, Banff, Canada, Jul. 2003. 